Discrete Comput Geom 34:111-128 (2005)

DOI: $10.1007 / \mathrm{s} 00454-005-1163-5$

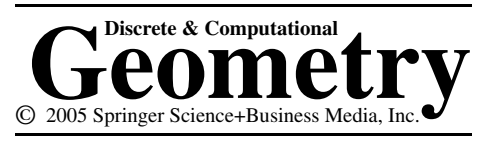

\title{
Configurations of Flats, I: Manifolds of Points in the Projective Line*
}

Jorge L. Arocha, Javier Bracho, and Luis Montejano

Instituto de Matemáticas, UNAM,

Ciudad Universitaria, México D.F. 04510

\{arocha,roli,luis\}@math.unam.mx

Dedicated to the memory of Victor Neumann-Lara

\begin{abstract}
The topological manifolds arising from configurations of points in the real and complex projective lines are classified. Their topology and combinatorics are described for the real case. A general setting for the study of the spaces of configurations of flats is established and a projective duality among them is proved in its full generality.
\end{abstract}

\section{Introduction}

This is the first paper in a series concerned with the topology and combinatorics of the spaces of configurations of flats in standard geometries. Configurations of vectors yield Grassmannians with a combinatorial stratification governed by matroids or oriented matroids [3]. Also, affine-configurations of points (i.e., $k$-tuples of points that affinely span modulo the affine group) yield Grassmannians but with a finer stratification governed by affine-oriented matroids. The motivation of this series is the fact that the topology of the space of transversals to convex sets is governed by the strata of the space of affine configurations of points (see [1] and [4]). The main question we raise is what happens when one considers flats other than zero-dimensional: what are the spaces so obtained and what are the combinatorics that govern their natural stratifications? The simplest new example to consider is configurations of lines in the real affine plane $\mathbb{A}^{2}$. As we will shortly see, the case of four lines is interesting enough to motivate the study of projective configurations of points.

* All authors were partially supported by Grants CONACYT-U41340-F and DGAPA-IN111702-3. 


\subsection{The First Example}

Consider two 4-tuples of lines $\ell_{1}, \ell_{2}, \ell_{3}, \ell_{4}$ and $\ell_{1}^{\prime}, \ell_{2}^{\prime}, \ell_{3}^{\prime}, \ell_{4}^{\prime}$ in the affine plane $\mathbb{A}^{2}$ as the same affine configuration, or to be affinely equivalent, if there is an affine isomorphism $f$ which maps one 4-tuple into the other, that is, $f\left(\ell_{i}\right)=\ell_{i}^{\prime}$. Since all contractions are affinities, then any affine configuration of lines is infinitely close to one where all the lines are concurrent; and then, furthermore, by contracting on another line, to the one where all coincide. So, one must rule out these degenerate configurations to obtain a "decent" topological space (for the case of vectors or points this was achieved by imposing the spanning hypothesis). The natural way to detect degeneracy is by the group action. We say that the 4-tuple of lines $\ell_{1}, \ell_{2}, \ell_{3}, \ell_{4}$ fixes the affine plane $\mathbb{A}^{2}$ if the only affine isomorphism $f$ which leaves the 4-tuple invariant, $f\left(\ell_{i}\right)=\ell_{i}$, is the identity. So, for example, if three of the four lines form a triangle, they fix; but if all are concurrent or three of them are parallel, they do not. Observe that fixing is a property of the affine configuration. So, the affine group acts freely in the set of 4-tuples of lines that fixes the plane and the quotient map is a principal bundle.

Let $L$ be the space of affine configurations of four lines in $\mathbb{A}^{2}$ that fix $\mathbb{A}^{2}$. The space $L$ is compact and locally homeomorphic to $\mathbb{R}^{2}$ but it is still not Hausdorff. Indeed, consider the sequence of 4-tuples of lines in Fig. 1. It clearly converges to the configuration shown in Fig. 1(a) $\left(\ell_{2}=\ell_{4}\right)$; but by appropriately expanding and contracting the two axis, it is easy to see that it is equivalent to sequences that converge to the configurations shown in Fig. 1(b) $\left(\ell_{1}=\ell_{3}\right)$ and Fig. 1(c) (two pairs of parallel lines: $\ell_{1} \| \ell_{3}$ and $\left.\ell_{2} \| \ell_{4}\right)$. So this sequence of configurations has (at least) three limiting points in $L$, which is therefore non-Hausdorff.

If we restrict, furthermore, to the configurations with four distinct lines, $L_{\neq}$say, that is, if we assume $\ell_{i} \neq \ell_{j}$ for $i \neq j$, then the sequence of Fig. 1 will "know" it converges to Fig. 1(c), and this is enough to obtain a nice compact Hausdorff space: the projective plane with four blow-ups, the surface of nonoriented genus 5 in Fig. 2 where the four inside circles and the boundary are antipodally identified.

This can be argued by means of charts. Let $U_{4} \subset L_{\neq}$consist of those configurations where $\ell_{1}, \ell_{2}, \ell_{3}$ form a triangle; which can then be assumed to be the standard one, so that $U_{4}$ is parametrized by $\ell_{4}$ and is therefore a projective plane with four punctures (in Fig. 2, think of the four inside circles as punctures, making them correspond to the line at

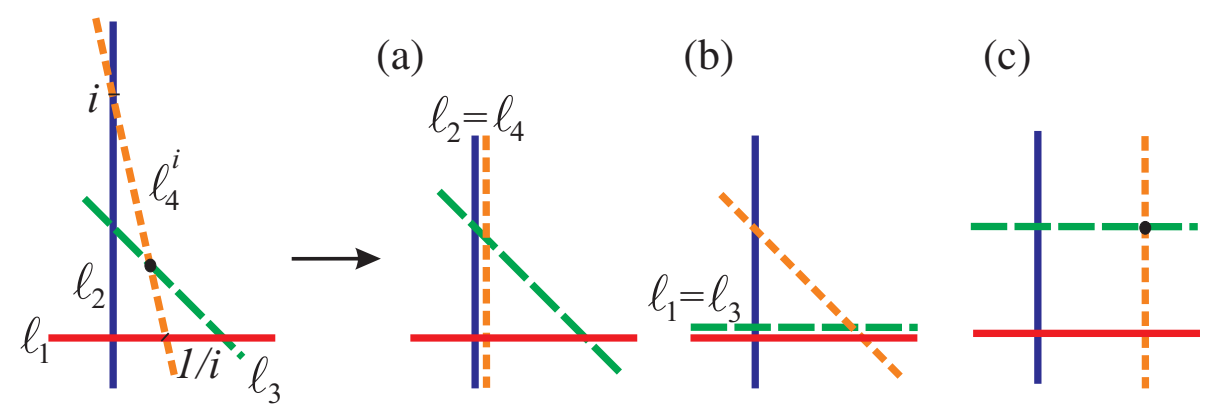

Fig. 1. A sequence of configurations of lines converging to three different configurations. 


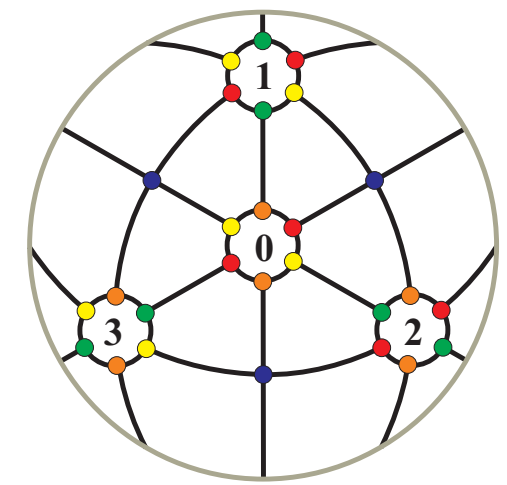

Fig. 2. The polyhedral complex of five different lines in $\mathbb{R} \mathbb{P}^{2}$.

infinity, labeled 0 , and to the three other lines $\ell_{i}$ to which $\ell_{4}$ is not allowed to be equal). The six drawn projective lines (with two punctures) correspond to three parallel pencils $\ell_{4} \| \ell_{i}$ (concurrent at the center), and three other pencils (forming the triangle) where $\ell_{4}$ is concurrent with two of the other lines. The three (dark) vertices that are covered by $U_{4}$ are then the three configurations: $\ell_{4}$ is parallel to $\ell_{i}$ and passes through $\ell_{j} \cap \ell_{k}$.

Changing the role of $\ell_{4}$ by the other three lines, we get four charts $U_{i}$ with the same picture (which cover $L_{\neq}$minus the three configurations of two pairs of parallel lines). In the new charts, we also see all the configurations in general position, but some of the punctures of $U_{4}$ appear as "nice" projective lines. The puncture 0 (when $\ell_{4}$ goes off to infinity) appears as the "nice" line where $\ell_{1}, \ell_{2}, \ell_{3}$ are concurrent in $U_{1}, U_{2}$, and $U_{3}$-because a 4-tuple where $\ell_{4}$ is far away (close to infinity) is the same configuration as a 4-tuple where $\ell_{4}$ is nearby and $\ell_{1}, \ell_{2}, \ell_{3}$ are almost concurrent. Analogously, one can see that, say, the puncture " $\ell_{3}=\ell_{4}$ ", labeled 3 in $U_{4}$, appears as the nice line " $\ell_{1} \| \ell_{2}$ " in $U_{1}$ and $U_{2}$.

At this point, it should be clear that our description problem is easier to address in the real projective plane $\mathbb{P}^{2}$, where a fifth line $\ell_{5}$ has been previously fixed "at infinity", and overlooked (our condition $\ell_{i} \neq \ell_{j}$ allows us to let it join the game), so that parallelism becomes just another case of three concurrent lines. Our charts can be redefined as "four of the lines are in general position," so that we get a new chart $U_{5}$ where our three missing vertices appear when $\ell_{5}$ is one of the three diagonals of a projective quadrilateral. Now, the symmetry of the combinatorial surface that arises from four different lines in the plane can be seen to grow to the symmetric group on five letters, $\mathfrak{S}_{5}$, and not only the expected $\mathfrak{S}_{4}$.

By classic duality (to which we refer as "polarity" in Section 4), we might as well think of projective configurations of five different points in $\mathbb{R} \mathbb{P}^{2}$. The 12 pentagonal 2-cells of Fig. 2 correspond to the dihedral orders (cyclic orders modulo orientation) that the unique conic through five points in general position imposes on them; edges and vertices correspond to degeneracies in the sense of colinearity.

Observe also that dihedral orders of five letters correspond to the general position cells of configurations of five points in the projective line $\mathbb{R P}^{1}$. In fact, Fig. 2 is also the space of projective configurations of five points in $\mathbb{R} \mathbb{P}^{1}$ with at least three of them different (the 
"spanning" or "fixing" hypothesis) and with no three of them equal (the "rule," which we will denote $\hat{3}$ ). Combinatorially, it is obtained from 12 pentagons with their vertices colored to match the 12 dihedral orders on five colors with two of them glued if they differ by a transposition (its natural generalization to obtain regular polyhedra for $n$ other than 5 is studied in [7]).

\subsection{Notation}

Let $\mathbb{K} \mathbb{P}^{n}=\left(\mathbb{K} \mathbb{P}^{n}, \operatorname{PGL}\left(\mathbb{K}^{n+1}\right)=\mathrm{GL}\left(\mathbb{K}^{n+1}\right) / \mathbb{K}^{*}\right)$ stand for the standard projective geometry over a field $\mathbb{K}$ (where we are mainly concerned with $\mathbb{K} \in\{\mathbb{R}, \mathbb{C}\}$ ). We say that a family of projective flats $F_{1}, F_{2}, \ldots, F_{k} \subset X$ fix if the unique $f \in \operatorname{PGL}\left(\mathbb{K}^{n}\right)$ that leaves them invariant $\left(f\left(F_{i}\right)=F_{i}\right)$ is the identity (the term arises because if we suppose they are fixed as subsets, then the space is pointwise fixed). Let $\mathbf{d}=\left(d_{1}, \ldots, d_{k}\right)$ be a vector of natural numbers. Then denote

$$
\mathbb{K P}_{\mathbf{d}}^{n}=\left\{\left(F_{1}, F_{2}, \ldots, F_{k}\right) \mid\left\{F_{1}, F_{2}, \ldots, F_{k}\right\} \text { fix and } \operatorname{dim} F_{i}=d_{i}\right\} / \operatorname{PGL}\left(\mathbb{K}^{n}\right),
$$

where the action of $\operatorname{PGL}\left(\mathbb{K}^{n}\right)$ is the natural free diagonal action $g\left(F_{1}, F_{2}, \ldots, F_{k}\right)=$ $\left(g\left(F_{1}\right), g\left(F_{2}\right), \ldots, g\left(F_{k}\right)\right)$. A PGL $\left(\mathbb{K}^{n}\right)$-equivalence class of a $k$-tuple of flats is a projective configuration.

For the affine geometry $\mathbb{K} \mathbb{A}^{n}=\left(\mathbb{K} \mathbb{A}^{n}, \operatorname{Aff}\left(\mathbb{K}^{n}\right)\right)$ the space of affine configurations $\mathbb{K} \mathbb{A}_{\mathbf{d}}^{n}$ is defined in the same way changing projective flats by affine flats and the group $\operatorname{PGL}\left(\mathbb{K}^{n}\right)$ by the affine group Aff $\left(\mathbb{K}^{n}\right)$.

We simplify our notation in three ways. First, we write $\mathbb{P}_{\mathbf{d}}^{n}=\mathbb{R} \mathbb{P}_{\mathbf{d}}^{n}$ and $\mathbb{A}_{\mathbf{d}}^{n}=\mathbb{R} \mathbb{A}_{\mathbf{d}}^{n}$. Second, if $\mathbf{d}=\left(d_{1}, \ldots, d_{k}\right)$ is homogeneous, i.e., $d_{1}=\cdots=d_{k}=d$, then we denote $\mathbb{K} \mathbb{P}_{\mathbf{d}}^{n}=\mathbb{K P}_{k, d}^{n}$. Third, if $d=0$, i.e., if the flats are points, then we denote $\mathbb{K} \mathbb{P}_{k, 0}^{n}=\mathbb{K} \mathbb{P}_{k}^{n}$

Moreover, if $\mathcal{R}$ is a "rule" specifying some allowed configurations, we denote by $\mathbb{K} \mathbb{P}_{\mathbf{d}}^{n}(\mathcal{R})$ the subspace of configurations that satisfy that rule. So, for example, $\mathbb{A}_{4,1}^{2}$ stands for $L$ above, and $\mathbb{A}_{4,1}^{2}(\neq)$ for four different lines in $\mathbb{A}^{2}$ that fix, which we were previously calling $L_{\neq}$. We can restate our previous considerations as $\mathbb{A}_{4,1}^{2}(\neq)=\mathbb{P}_{5,1}^{2}(\neq)=\mathbb{P}_{5}^{2}(\neq)$; and we will further have $\mathbb{P}_{5}^{2}(\neq) \cong \mathbb{P}_{5}^{1}(\hat{3})$ (where $\hat{3}$ stands for the rule "no three elements of the configuration are equal"), because of a general duality among projective configurations which we establish in Section 4.

\subsection{Quirurgical Manifolds}

As should be clear from the example above, configuration spaces of flats can be nonHausdorff, because we are dividing by noncompact Lie groups. However, they seem to have a beauty of their own. We will prove that $\mathbb{P}_{k}^{1}$ has the structure of what can be called a quirurgical manifold.

Classic surgery arises from the fact that if $r+t=n-1$, then the standard embeddings $S^{r} \hookrightarrow S^{n}$ and $S^{t} \hookrightarrow S^{n}$ have trivial normal bundles and their regular neighborhoods can be made to match their boundaries:

$$
S^{n}=\partial\left(B^{r+1} \times B^{t+1}\right)=S^{r} \times B^{t+1} \underset{S^{r} \times S^{t}}{\cup} B^{r+1} \times S^{t} .
$$


So that a sphere $S^{r}$ with a trivial normal bundle in a manifold $M^{n}$ can be cut out and replaced by a sphere of complementary dimension $S^{t}$. This procedure can be thought of as taking out an open regular neighborhood of $S^{r}$ and then sewing back the regular neighborhood of $S^{t}$ (in $S^{n}$ ), because the boundaries coincide. However, it can also be thought of, and some authors do, as taking only the core $S^{r}$ out and sewing in the alternative core $S^{t}$, because, furthermore, their regular neighborhoods (in $S^{n}$ ) without their zero sections (we call them their punctured regular neighborhoods) are homeomorphic. If we think of both cores simultaneously sewn in, we have a "quirurgical manifold."

More precisely, we have a homeomorphism

$$
\begin{aligned}
h: S^{r} \times B^{t+1} \backslash S^{r} & \times\{0\} \rightarrow B^{r+1} \times S^{t} \backslash\{0\} \times S^{t}, \\
h(x, y) & =\left(|y| x, \frac{y}{|y|}\right),
\end{aligned}
$$

with inverse $h^{-1}(x, y)=\left(|x|^{-1} x,|x| y\right)$. We define the quirurgical torus to be

$$
H^{r, t}=S^{r} \times B^{t+1} \cup_{h} B^{r+1} \times S^{t},
$$

that is, on the disjoint union identify $(x, y) \sim h(x, y)$ for all $(x, y) \in S^{r} \times B^{t+1} \backslash S^{r} \times\{0\}$, and we call $S^{r} \times\{0\}$ and $\{0\} \times S^{t}$ the nearby cores, $S^{r}$ and $S^{t}$, of $H^{r, t}$. At them, $H^{r, t}$ fails to be Hausdorff, but from any point of one core one can go to any point on the other through two infinitesimal displacements in the appropriate directions.

Let $\overrightarrow{\mathbb{P}}{ }_{k}^{1}$ be the space of oriented projective configurations of $k$-points in $\mathbb{P}^{1}$ that fix (we only mod out by orientation-preserving projectivities), so that $\overrightarrow{\mathbb{P}}_{k}^{1}$ is a double cover of $\mathbb{P}_{k}^{1}$. Since a set of points fixes $\mathbb{P}^{1}$ if and only if three of them are different, both spaces are "non-Hausdorff manifolds" of dimension $k-3$. We will prove that $\overrightarrow{\mathbb{P}}_{k}^{1}$ is compact and has a finite family of embedded full-dimensional quirurgical tori $H^{r, t}(r+t=k-4)$ such that the non-Hausdorff phenomena only occurs at their cores. More precisely, if $x$ and $y$ are (non-Hausdorffly) attached (i.e., without disjoint neighborhoods), then they belong to cores which are either complementary (nearby cores of the same quirurgical torus) or such that their complementary cores intersect. Moreover, we will see that the orientation involution acts on each of these quirurgical tori as the antipodal map on $S^{k-3}$. would. So that in $\mathbb{P}_{k}^{1}$ we have pairs of complementary projective flats $\mathbb{P}^{r}$ and $\mathbb{P}^{t}$ (with $r+t=k-4$ ), the nearby cores, sharing punctured regular neighborhoods (i.e., without the zero-section) homeomorphic to, and with the bundle structure of, their standard regular neighborhoods in $\mathbb{P}^{k-3}$.

To fix ideas, we can now complete the description of $\mathbb{P}_{5}^{1}$ (and $\mathbb{P}_{5,1}^{2}$ ): in Fig. 2, we are only missing ten new points, $\mathbb{P}^{0}$, each of them associated as nearby cores to one of the ten dark lines, $\mathbb{P}^{1}$, and therefore sharing their punctured regular neighborhoods. Observe that, indeed, the ten dark lines of Fig. 2 have a Möbius band as the regular neighborhood, which if punctured (the core taken out) are naturally homeomorphic to a disk minus a point. So that, for example, thinking again in terms of five lines in $\mathbb{P}^{2}$, that is, in $\mathbb{P}_{5,1}^{2}$, the unique configuration where two lines are the same, say $\ell_{4}=\ell_{5}$, is the nearby core, $\mathbb{P}^{0}$, to the line, $\mathbb{P}^{1}$, of configurations where the other three lines are concurrent, $\ell_{1} \cap \ell_{2} \cap \ell_{3} \neq \emptyset$. Then, e.g., for the rule $\mathcal{F}_{4}$ ("free 4 ") where $\ell_{4}$ can do whatever it pleases but three lines 
cannot meet at a point unless $\ell_{4}$ is one of them, we have that $\mathbb{P}_{5,1}^{2}\left(\mathcal{F}_{4}\right) \cong \mathbb{P}^{2}$, because the four inside (circular) lines of Fig. 2 are replaced by surgery with their corresponding $\mathbb{P}^{0}$ nearby cores.

To complete our description of four lines in $\mathbb{A}^{2}$, observe that $\mathbb{A}_{4,1}^{2}$ is obtained by deleting from $\mathbb{P}_{5,1}^{2}$, four of the ten special points, corresponding to configurations where a line (the one chosen to be at infinity) is equal to one of the other four. In our original description of Fig. 2 as $U_{4}$, and $\ell_{5}$ at infinity, they correspond to the $\mathbb{P}^{0}$ nearby cores of the (circular) line at the center and the three (nondiameter) lines forming a triangle; but in $U_{5}$, the deleted four points $\left(\ell_{5}=\ell_{i}\right)$ would correspond to the inside circles.

\subsection{The Program}

After the motivating examples which should increase the intuition of the reader, we finish the Introduction with a brief description of the paper.

Section 2 is the core of the paper. There, we characterize the rules for which $\mathbb{K P}_{k}^{1}(\mathcal{R})$ is a compact Hausdorff manifold $(\mathbb{K} \in\{\mathbb{R}, \mathbb{C}\})$. Moreover, it is proved that all such manifolds of "full" dimension contained in $\mathbb{K P}_{k}^{1}$ arise in this way and that there are a finite number of them.

Although some of these results can be generalized to dimensions higher than 1, the dimension 1 case is specially interesting because the simplicity of the object of study makes it possible to reveal facts which are not true in higher dimensions. Therefore, in Section 3 we describe the stratification of $\mathbb{R P}_{k}^{1}$ governed by dihedral partitions, in particular, it gives the structure of a polyhedral complex to the manifolds $\mathbb{R P}_{k}^{1}(\mathcal{R})$. Moreover, all manifolds $\mathbb{R P}_{k}^{1}(\mathcal{R})$ can be obtained from each other by projective surgery and this shows that $\mathbb{R P}_{k}^{1}$ is a "quirurgical manifold" in the sense above.

In Section 4 we show the existence of a natural homeomorphism $\mathbb{K} \mathbb{P}_{\mathbf{d}}^{n} \simeq \mathbb{K P}_{\mathbf{d}}^{m}$ for any field $\mathbb{K}$. It is a generalization for configurations of projective flats of the well-known (see [6]) duality between configurations of projective points. This allow us to reveal the structure of many other spaces of projective configurations and, in particular, to formalize the homeomorphism $\mathbb{P}_{5}^{1} \simeq \mathbb{P}_{5}^{2}$ used above.

\section{The Topology of $\mathbb{K P}_{k}^{1}$}

In this section $\mathbb{K} \in\{\mathbb{R}, \mathbb{C}\}$, in particular, it is a metric field of characteristic 0 such that $\mathbb{K} \mathbb{P}^{n}$ is a compact manifold. We do not use the complex number $i$, so we will use this letter for general purposes. By $\infty$ we denote the point added to $\mathbb{R}\left(\right.$ or $\mathbb{C}$ ) to obtain $\mathbb{R}^{1}$ ( or $\mathbb{C P}^{1}$ ). We use $\|p\|$ to denote the distance to 0 of the projective point $p$. We emphasize that all dimensions are computed over $\mathbb{K}$. So, a complex projective line has dimension 1 .

\subsection{Blocks and Rules}

Let $\mathbf{p}=\left(p_{1}, \ldots, p_{k}\right)$ be a $k$-tuple of points in the projective space $\mathbb{K} \mathbb{P}^{n}$, i.e., $\mathbf{p} \in$ $\left(\mathbb{K} \mathbb{P}^{n}\right)^{k}$. We denote by $[\mathbf{p}]=\left[p_{1}, \ldots, p_{k}\right]$ its equivalence class modulo the projective 
group PGL $\left(\mathbb{K}^{n+1}\right)=\mathrm{GL}\left(\mathbb{K}^{n+1}\right) / \mathbb{K}^{*}$, and call it a projective configuration, or simply a configuration, when $p_{1}, \ldots, p_{k}$ fix $\mathbb{K} \mathbb{P}^{n}$; otherwise we may call it a degenerate configuration. The space of configurations of $k$ points in $\mathbb{K P}^{n}$, which we have called $\mathbb{K P}_{k}^{n}$, is then the quotient of an open subspace of $\left(\mathbb{K} \mathbb{P}^{n}\right)^{k}$ (the fixing $k$-tuples) over $\operatorname{PGL}\left(\mathbb{K}^{n}\right)$.

Let $A$ be a subset of indices. We denote $\mathbf{p}_{A}:=\left\{p_{i}: i \in A\right\}$. Let $\alpha \in\{0, \ldots, n-1\}$, we say that $[\mathbf{p}]$, or $\mathbf{p}$, has block $A_{\alpha}$ if $\operatorname{dim}\left\langle\mathbf{p}_{A}\right\rangle \leq \alpha$, this is, if the dimension of the projective subspace spanned by $\mathbf{p}_{A}$ is not greater than $\alpha$. Observe that $\mathbf{p}$ has block $A_{\alpha}$ for every $\alpha \geq \sharp A-1$, but having block $A_{\alpha}$ for small $\alpha$ detects some "dimensional" degeneracy of the configuration.

A rule $\mathcal{R}$ is a subset of all possible blocks, called the permitted ones so that its complement consists of the prohibited blocks. Denote by $\mathbb{K P}_{k}^{n}(\mathcal{R})$ the space of all configurations such that all its blocks are permitted (are in $\mathcal{R}$ ).

For the projective line, $n=1$, the subindex $\alpha$ is always zero and the fact that a configuration $[\mathbf{p}]=\left[p_{1}, \ldots, p_{k}\right]$ has block $A_{0}$ means that $p_{i}=p_{j}, \forall i, j \in A$, so we may simplify our notation to say that " $[\mathbf{p}]$, or $\mathbf{p}$, has block $A$." For a block $A$ we denote the complement of $A$ by $\bar{A}$.

Since our main interest are the spaces $\mathbb{K P}_{k}^{1}(\mathcal{R})$, we further assume that a rule $\mathcal{R}$ satisfies:

R1. The blocks $\{i\}$ are permitted and the blocks $\overline{\{i\}}$ are prohibited.

R2. If $B \in \mathcal{R}$ and $A \subseteq B$, then $A \in \mathcal{R}$ (subsets of permitted blocks are permitted).

Because a permitted block with a prohibited subblock would have no occurrence, we might as well declare it prohibited; a block $\overline{\{i\}}$ cannot appear in a fixing configuration so we can also declare it prohibited; and if $\{i\}$ is prohibited, then $\mathbb{K P}_{k}^{1}(\mathcal{R})$ is void. So for any rule $\mathcal{R}$ we now have that $\mathbb{K P}_{k}^{1}(\mathcal{R})$ contains all configurations in general position whose only blocks are the singletons $\{i\}$.

We shall prove the following theorem, where, as usual, a manifold is assumed to be Hausdorff.

Theorem 2.1. A subspace $\mathcal{T} \subset \mathbb{K P}_{k}^{1}$ is a compact closed manifold of full dimension $\left(\operatorname{dim} \mathcal{T}=\operatorname{dim} \mathbb{K} \mathbb{P}_{k}^{1}=k-3\right)$ if and only if $\mathcal{T}=\mathbb{K} \mathbb{P}_{k}^{1}(\mathcal{R})$ for a rule $\mathcal{R}$ that satisfies:

R3. $A \in \mathcal{R} \Leftrightarrow \bar{A} \notin \mathcal{R}$,

where $\bar{A}$ denotes the complement of $A$.

We remark, before we go into the technicalities, that $\mathbb{K} \mathbb{P}_{k}^{1}$ is locally homeomorphic to $\mathbb{K}^{k-3}$. Observe that a $k$-tuple $\mathbf{p}=\left(p_{1}, \ldots, p_{k}\right) \in\left(\mathbb{K} \mathbb{P}^{1}\right)^{k}$ fixes $\mathbb{K} \mathbb{P}^{1}$ if and only if it has at least three different points. Then a small neighborhood of a configuration [p] in $\mathbb{K P}_{k}^{1}$ can be obtained by keeping three different points in $\mathbf{p}$ constant and moving its remaining $k-3$ points in small neighborhoods (locally homeomorphic to $\mathbb{K}$ ) around them. The problem that makes Theorem 2.1 interesting is that compactness is not obvious and that $\mathbb{K P}_{k}^{1}$ is not Hausdorff. 


\subsection{The Compactification of $\operatorname{PGL}\left(\mathbb{K}^{2}\right)$}

We say that a sequence of projectivities $f^{i} \in \mathrm{PGL}\left(\mathbb{K}^{2}\right)$ converges point to point if for any $x \in \mathbb{K} \mathbb{P}^{1}$, the sequence $f^{i}(x)$ has a limit in $\mathbb{K} \mathbb{P}^{1}$; if this is the case, the sequence $f^{i}$ has a limit function $\lim f^{i}: x \mapsto \lim f^{i}(x)$. Since the group PGL $\left(\mathbb{K}^{2}\right)$ is not compact, there are sequences of projectivities that converge point to point but do not converge in $\operatorname{PGL}\left(\mathbb{K}^{2}\right)$, in others words, the function $\lim f^{i}$ need not be a projectivity. Let $\overline{\operatorname{PGL}}\left(\mathbb{K}^{2}\right)$ be the set of all limits of sequences of projectivities that converge point to point. We clearly have that if $f \in \overline{\operatorname{PGL}}\left(\mathbb{K}^{2}\right)$ and $g \in \operatorname{PGL}\left(\mathbb{K}^{2}\right)$, then $f \circ g, g \circ f \in \overline{\operatorname{PGL}}\left(\mathbb{K}^{2}\right)$.

For an example of a function in $\overline{\mathrm{PGL}}\left(\mathbb{K}^{2}\right) \backslash \mathrm{PGL}\left(\mathbb{K}^{2}\right)$ consider the sequence $f^{i}(x)=i x$ whose limit is the function

$$
\pi_{0, \infty}: x \mapsto\left\{\begin{array}{lll}
0 & \text { if } & x=0, \\
\infty & \text { if } & x \neq 0,
\end{array}\right.
$$

which is called the projection from 0 to $\infty$. Another example is the sequence $f^{i}(x)=$ $i(x+i)$ whose limit maps any point to $\infty$; it is the constant function $\pi_{\infty}$.

These two examples are universal in the following sense. Two functions $f, g \in$ $\overline{\mathrm{PGL}}\left(\mathbb{K}^{2}\right)$ are said to be projectively equivalent if there exist projectivities $h, h^{\prime} \in$ $\operatorname{PGL}\left(\mathbb{K}^{2}\right)$ such that $f \circ h=h^{\prime} \circ g$.

Proposition 2.2. Any function in $\overline{\mathrm{PGL}}\left(\mathbb{K}^{2}\right)$ is projectively equivalent either to the identity map, to $\pi_{\infty}$, or to $\pi_{0, \infty}$.

Proof. Let $f=\lim f^{i}$ with $f^{i} \in \operatorname{PGL}\left(\mathbb{K}^{2}\right)$. Projectivities are $2 \times 2$ nonsingular matrices modulo nonzero elements of $\mathbb{K}$. Therefore $\operatorname{PGL}\left(\mathbb{K}^{2}\right)$ is contained in the space of $2 \times 2$ nonzero matrices modulo $\mathbb{K}^{*}$, that is $\operatorname{PGL}\left(\mathbb{K}^{2}\right) \subset \mathbb{K} \mathbb{P}^{4}$. Since $\mathbb{K} \mathbb{P}^{4}$ is compact, there must exist a $2 \times 2$ nonzero matrix $A$ with entries in $\mathbb{K}$ such that

$$
\forall x \notin \operatorname{Ker} A, \quad f(x)=A x \bmod \mathbb{K}^{*} .
$$

If $A$ has rank 2 , then $f$ is a projectivity and is projectively equivalent to the identity map

If rank $A=1$, then the subspaces $q=\operatorname{Im} A$ and $p=\operatorname{Ker} A$ are lines in $\mathbb{K}^{2}$, i.e., points in $\mathbb{K P}^{1}$. If $f(p)=q$, then $f$ is a constant function to $q$ and is projectively equivalent to $\pi_{\infty}$

Suppose $f(p) \neq q$. Let $g$ be any projectivity that maps 0 to $p$ and let $h$ be any projectivity that maps $f(p)$ to 0 and $q$ to $\infty$. The function $h \circ f \circ g$ is equal to $\pi_{0, \infty}$. $\square$

Remark 2.1. Any sequence $f^{i}$ in $\operatorname{PGL}\left(\mathbb{K}^{2}\right)$ has a point to point converging subsequence. Suppose $f=\lim f^{i}$ is not given in the preceding proof. The sequence of "matrices" in $\mathbb{K} \mathbb{P}^{4}$ can still be constructed, and a converging subsequence, to $A$, extracted. Then, in the final step for a projection, a subsequence such that $f^{i}(p)$ converges can be obtained because $\mathbb{K} \mathbb{P}^{1}$ is compact.

Remark 2.2. Let $f^{i}$ be a point to point converging sequence of projectivities and let $x^{i}$ be a converging sequence of points in $\mathbb{K} \mathbb{P}^{1}$; denote $f=\lim f^{i}$ and $x=\lim x^{i}$. Then 
we have that $\lim f^{i}\left(x^{i}\right)=f(x)$ unless $f$ is the projection from $p$ to $q, f=\pi_{p, q}$, and $x=p$, because, otherwise, $f$ is continuous at $x$. In the noncontinuous case, $f=\pi_{p, q}$ and $x=p$, we have that $\lim f^{i}\left(x^{i}\right)$ depends on the "speeds" of convergence of $f^{i}$ and $x^{i}$, and $\lim f^{i}\left(x^{i}\right)$ can be anything (consider the sequence $x^{i}=y / i$ in our definition of $\left.\pi_{0, \infty}\right)$.

\subsection{Attached Configurations}

Two configurations [p], [q] $\in \mathbb{K P}_{k}^{1}$ are said to be (non-Hausdorffly) attached if they are different and there are two projectively equivalent sequences of fixing $k$-tuples $\mathbf{p}^{i}, \mathbf{q}^{i}$ such that $\lim _{i \rightarrow \infty} \mathbf{p}^{i}=\mathbf{p}$ and $\lim _{i \rightarrow \infty} \mathbf{q}^{i}=\mathbf{q}$. We say that $[\mathbf{p}]$ has a maximal block $A$ if it has block $A$ but does not have block $A^{\prime}$ for any superset $A^{\prime} \supset A$.

Proposition 2.3. The configurations $[\mathbf{p}]$ and $[\mathbf{q}]$ in $\mathbb{K} \mathbb{P}_{k}^{1}$ are attached if and only if there exists a subset $A$ of indices, such that $[\mathbf{p}]$ has maximal block $A$ and $[\mathbf{q}]$ has block $\bar{A}$.

Proof. Let $\mathbf{p}^{i}$ and $\mathbf{q}^{i}$ be the projectively equivalent sequences of fixing $k$-tuples whose limits are $\mathbf{p}$ and $\mathbf{q}$, respectively. Let $f^{i}$ be the projectivity such that $f^{i}\left(\mathbf{p}^{i}\right)=\mathbf{q}^{i}$. By Remark 2.1, we may assume $f^{i}$ converges point to point, so let $f=\lim f^{i} \in \overline{\operatorname{PGL}}\left(\mathbb{K}^{2}\right)$. If $f$ is a projectivity, then $[\mathbf{p}]=[\mathbf{q}]$, and if it is a constant function, then $\mathbf{q}$ does not fix by Remark 2.2; so it has to be a projection and we may assume that $f=\pi_{0, \infty}$ by Proposition 2.2. Let $A\left\{j: p_{j}=0\right\}$, so that [p] has maximal block $A$. If $j \notin A$, then, by Remark 2.2,

$$
q_{j}=\lim f^{i}\left(p_{j}^{i}\right)=\pi_{0, \infty}\left(p_{j}\right)=\infty .
$$

This proves that q has block $\bar{A}$.

Conversely, suppose $[\mathbf{p}]=\left[p_{1}, \ldots, p_{k}\right],[\mathbf{q}]=\left[q_{1}, \ldots, q_{k}\right] \in \mathbb{K} \mathbb{P}_{k}^{1}$ are configurations such that for the subset $A$ of indices, [p] has maximal block $A$ and [q] has block $\bar{A}$. They must be different configurations because each has at least three different points. Let $B$ be the maximal block of [q] containing $\bar{A}$. We may assume that $\mathbf{p}_{A}=\{0\}$ and $\mathbf{q}_{B}=\{\infty\}$. Let $\mathbf{p}^{i}=\left(p_{1}^{i}, \ldots, p_{k}^{i}\right)$ be the sequence

$$
p_{j}^{i}= \begin{cases}p_{j}, & j \in \bar{A}, \\ 1 / i, & j \in A \cap B, \\ \left(1 / i^{2}\right) q_{j}, & j \in \bar{B},\end{cases}
$$

and let $f^{i}(x)=i^{2} x$. Observe that all (but at most one) of the $\mathbf{p}^{i}$ fix. Clearly, $\mathbf{p}=\lim \mathbf{p}^{i}$ and $\mathbf{q}=\lim f^{i}\left(\mathbf{p}^{i}\right)$, proving that $[\mathbf{p}]$ and $[\mathbf{q}]$ are attached.

\subsection{The Submanifolds of $\mathbb{K P}_{k}^{1}$}

We are now in a position to prove Theorem 2.1. First, we prove the part that classifies the rules that yield compact manifolds. 
Proposition 2.4. Let $\mathcal{R}$ be a rule (satisfying $\mathrm{R} 1$ and $\mathrm{R} 2$ ). Then $\mathbb{K P}_{k}^{1}(\mathcal{R})$ is a compact closed manifold if and only if $\mathcal{R}$ satisfies:

R3. $A \in \mathcal{R} \Leftrightarrow \bar{A} \notin \mathcal{R}$.

Proof. Let $[\mathbf{p}] \in \mathbb{K P}_{k}^{1}(\mathcal{R})$. Let $\varepsilon$ be a neighborhood of $[\mathbf{p}]$ in $\mathbb{K} \mathbb{P}_{k}^{1}$. If $p_{i} \neq p_{j}$ and $\varepsilon$ is small enough, then $p_{i} \neq p_{j}$ also holds for any configuration in $\varepsilon$. Hence, $\varepsilon \subset \mathbb{K P}_{k}^{1}(\mathcal{R})$ and therefore $\mathbb{K P}_{k}^{1}(\mathcal{R})$ is locally homeomorphic to $\mathbb{K}^{k-3}$.

If $\mathbb{K P}_{k}^{1}(\mathcal{R})$ is not Hausdorff, then it contains two attached configurations and, by Proposition 2.3, there is $A$ such that $A, \bar{A} \in \mathcal{R}$. Reciprocally, suppose there is $A$ such that $A, \bar{A} \in \mathcal{R}$. Let $\left\{x_{1}, \ldots, x_{k}\right\}$ be a set of $k$ different projective points not intersecting $\{0, \infty\}$. Let $\mathbf{p}^{i}\left(p_{1}^{i}, \ldots, p_{k}^{i}\right)$ be the sequence

$$
p_{j}^{i}= \begin{cases}x_{j}, & j \in A \\ i x_{j}, & j \in \bar{A}\end{cases}
$$

where all but a finite number of the $\mathbf{p}^{i}$ are in general position. By R1, $\# \bar{A}<k-1$ and therefore $\mathbf{p}=\lim \mathbf{p}^{i}$ fixes. Since $\mathbf{p}$ has one maximal nontrivial block $\bar{A}$, we have $[\mathbf{p}] \in \mathbb{K P}_{k}^{1}(\mathcal{R})$. The same arguments give that $[\mathbf{q}]=\left[\lim \mathbf{p}^{i} / i\right]$ belongs to $\mathbb{K P}_{k}^{1}(\mathcal{R})$. Since $[\mathbf{p}] \neq[\mathbf{q}]$ we conclude that $\mathbb{K P}_{k}^{1}(\mathcal{R})$ is not Hausdorff.

We proved that $\mathbb{K P}_{k}^{1}(\mathcal{R})$ is Hausdorff iff $A \in \mathcal{R} \Rightarrow \bar{A} \notin \mathcal{R}$. So that we are only left to worry about the proof that $\mathbb{K P}_{k}^{1}(\mathcal{R})$ is compact iff $A \in \mathcal{R} \Leftarrow \bar{A} \notin \mathcal{R}$.

Suppose there is an $A$ such that $A, \bar{A} \notin \mathcal{R}$. It is easy to construct a sequence $\left[\mathbf{p}^{i}\right] \rightarrow[\mathbf{p}]$ such that all $\mathbf{p}^{i}$ are in general position and $\mathbf{p}$ has maximal block $A$. By Proposition 2.3 all configurations attached to $[\mathbf{p}]$ have the block $\bar{A}$. Therefore $\left[\mathbf{p}^{i}\right]$ does not converge in $\mathbb{K P}_{k}^{1}(\mathcal{R})$ and it is not compact

Reciprocally, suppose that $A \in \mathcal{R} \Leftarrow \bar{A} \notin \mathcal{R}$ and let $\left[\mathbf{p}^{i}\right] \in \mathbb{K P}_{k}^{1}(\mathcal{R})$ be a sequence of configurations. Since it is easy to see that $\mathbb{K} \mathbb{P}_{k}^{1}$ is compact, there is $[\mathbf{p}]=\lim \left[\mathbf{p}^{i}\right] \in \mathbb{K} \mathbb{P}_{k}^{1}$. If $[\mathbf{p}] \in \mathbb{K P}_{k}^{1}(\mathcal{R})$, then we are done. If not, then [p] has a prohibited maximal block, $A$ say, and therefore $\bar{A} \in \mathcal{R}$.

We may clearly assume that $\mathbf{p}_{A}=\{0\}$, and also that $\infty \notin \mathbf{p}_{A}^{i}$ because $\mathbf{p}_{A}^{i}$ tends to $\{0\}$. We need a sequence $\mathbf{q}^{i}$ projectively equivalent to $\mathbf{p}^{i}$ with limit $\mathbf{q}$ such that $\mathbf{q}_{\bar{A}}=\{\infty\}$ and $\{0,1\} \subset \mathbf{q}_{A} \subset B_{1}=\{x \mid 1 \geq\|x\|\}$ (the closed unitary ball). This can be achieved by choosing $v^{i}=p_{j}^{i}$ such that $\left\|p_{j}^{i}\right\|=\min _{\ell \in A}\left\|p_{\ell}^{i}\right\|$ and $\mu^{i}=p_{j}^{i}$ at maximum distance from $v^{i}$. Since $\mathbf{p}^{i}$ has all its blocks permitted, we have that $\sharp \mathbf{p}_{A}^{i} \geq 2$ and hence for each $i$ these points are different and well defined. Let $f^{i} \in \operatorname{PGL}\left(\mathbb{K}^{2}\right)$ be the projectivity defined by $f^{i}\left(\nu^{i}\right)=0, f^{i}\left(\mu^{i}\right)=1$, and $f^{i}(\infty)=\infty$, and $\mathbf{q}^{i}:=f^{i}\left(\mathbf{p}^{i}\right)$.

Since $\mathbf{q}=\lim \mathbf{q}^{i}$ contains 0,1 , and $\infty$, it fixes. If $\sharp A=2$, then $\mathbf{q}$ has maximal blocks $\bar{A}($ at $\infty)$ and singletons at 0 and 1 ; therefore, $[\mathbf{q}] \in \mathbb{K P}_{k}^{1}(\mathcal{R})$ because all of its blocks are permitted. If $[\mathbf{q}] \notin \mathbb{K} \mathbb{P}_{k}^{1}(\mathcal{R})$, then $\mathbf{q}$ has a maximal prohibited block $A^{\prime}$, but with $A^{\prime}$ a strict subset of $A\left(2 \leq \sharp A^{\prime}<\sharp A\right)$, and we may repeat this process (taking $\mathbf{p}=\mathbf{q}, \mathbf{p}^{i}=\mathbf{q}^{i}$ and $A=A^{\prime}$ ) to obtain, after a finite number of iterations, a limiting configuration in $\mathbb{K P}_{k}^{1}(\mathcal{R})$.

Now, let us complete the proof of Theorem 2.1. 
Proof of Theorem 2.1. Observe that the if side of the theorem follows from the preceding result. So, suppose $\mathcal{T} \subset \mathbb{K P}_{k}^{1}$ is a compact closed (Hausdorff) manifold of full dimension. Let $\mathcal{R}$ be the set of subsets of indices $A$ such that there exists some $[\mathbf{p}] \in \mathcal{T}$ with $A$ a block of $[\mathbf{p}]$. Then, since an open set in $\mathcal{T}$ is also open in $\mathbb{K P}_{k}^{1}$ (the full dimensionality) and any neighborhood of any point in $\mathbb{K P}_{k}^{1}$ contains configurations in general position, $\mathcal{R}$ contains all the singletons and so it satisfies $\mathrm{R} 1$. It obviously satisfies $\mathrm{R} 2$. If it does not satisfy $\mathrm{R} 3$, then there would exist $[\mathbf{p}],[\mathbf{q}] \in \mathcal{T}$ such that $[\mathbf{p}]$ has block $A$ and [q] has block $\bar{A}$. Since both configurations fix we have that $2 \leq \sharp A \leq k-2$. Then, by Proposition 2.3, any two neighborhoods in $\mathcal{T}$ of $[\mathbf{p}]$ and [q] would intersect, implying $\mathcal{T}$ is not Hausdorff. So $\mathcal{R}$ also satisfies R3.

By definition of $\mathcal{R}$, it is clear that $\mathcal{T} \subset \mathbb{K P}_{k}^{1}(\mathcal{R})$. Since, by hypothesis and Proposition 2.4, both are closed compact manifolds of the same dimension, then they must be equal.

\section{Combinatorial and Quirurgical Structure of $\mathbb{P}_{k}^{1}$}

In this section we restrict ourself to the case of the field of real numbers. First, we describe a polyhedral decomposition of the manifolds $\mathbb{P}_{k}^{1}(\mathcal{R})=\mathbb{R P}_{k}^{1}(\mathcal{R})$ and then we study the quirurgical structure of $\mathbb{R} \mathbb{P}_{k}^{1}$. A similar study for the case of the field of complex numbers will require further research.

\subsection{Dihedral Partitions and Polyhedral Complexes}

An ordered partition of a finite set $A$ is an $m$-tuple $\left(A_{1}, A_{2}, \cdots, A_{m}\right)$ of disjoint subsets, called blocks, such that $A_{1} \dot{\cup} A_{2} \dot{\cup} \ldots \dot{\cup} A_{m}=A$. A linear partition is an equivalence class of ordered partitions modulo the orientation involution (there are two possible orders or orientations for a linear partition); a cyclic partition is an equivalence class of ordered partitions modulo the corresponding cyclic group, and a dihedral partition is an equivalence class modulo the natural action of the dihedral group (the two cyclic partitions which are the same dihedral partition are its two possible orientations). One should think of a dihedral partition as a necklace of subsets of $A$.

Dihedral partitions (like the other kinds) have a natural partial order. Indeed, if in a necklace of subsets we join two consecutive blocks we obtain another dihedral partition coarser (or smaller) than the original. The Hasse diagram of the dihedral partitions of $\{1,2,3,4\}$ is shown in Fig. 3 .

We denote the poset (partially ordered set) of dihedral partitions of $\{1, \ldots, k\}$ by $\mathcal{D}_{k}$ with partial order $\preceq$. It has a rank function $\alpha \mapsto|\alpha|$, the number of subsets in the dihedral partition $\alpha$. The maximal elements in $\mathcal{D}_{k}$ are called dihedral orders, they are permutations modulo the action of the dihedral group.

Each configuration $[\mathbf{p}] \in \mathbb{P}_{k}^{1}$ has naturally associated a dihedral partition $d_{\mathbf{p}} \in \mathcal{D}_{k}$, called the type of [p], given by the dihedral order of its maximal blocks (which look like points) along $\mathbb{P}^{1}$ (which is a circle). Observe that since configurations have, by the fixing hypothesis, at least three points, then all their types have at least rank $3\left(\left|d_{\mathbf{p}}\right| \geq 3\right)$, so, abusing notation, we denote also by $\mathcal{D}_{k}$ the poset of dihedral orders with rank at least 3 . 


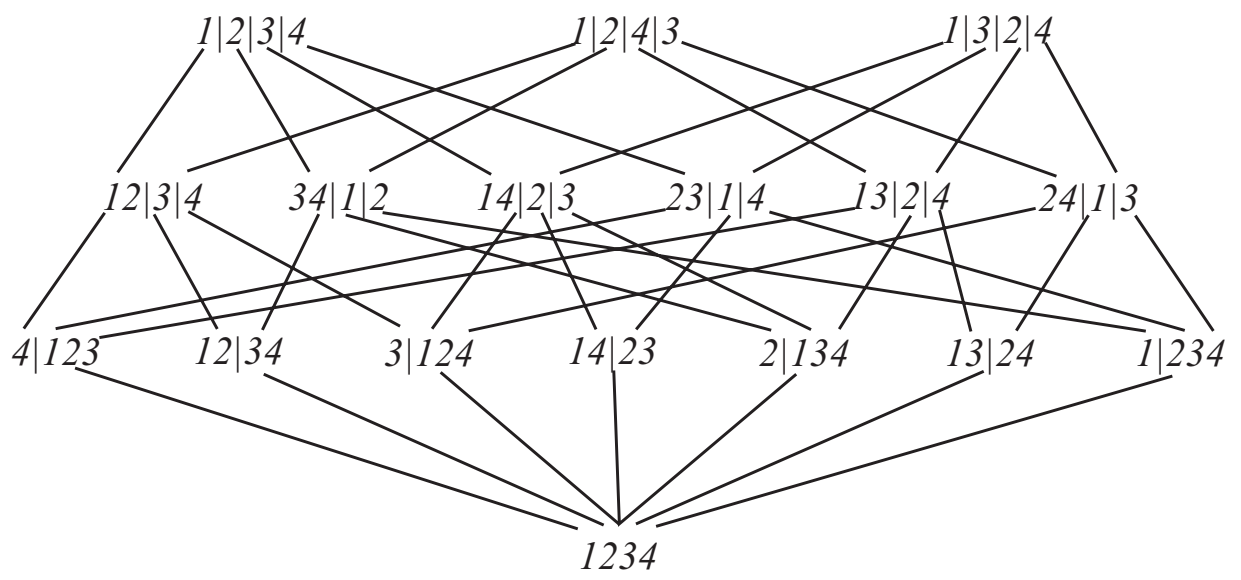

Fig. 3. The poset of dihedral partitions of $\{1,2,3,4\}$.

Lemma 3.1. Given $\alpha \in \mathcal{D}_{k}$ (with $|\alpha| \geq 3$ ), the set of all configurations $[\mathbf{p}] \in \mathbb{P}_{k}^{1}$ such that $d_{\mathbf{p}}=\alpha$ is an open ball of dimension $|\alpha|-3$, which we denote $\sigma_{\alpha}$ and call the cell of $\alpha$.

Proof. The dihedral partition $\alpha$ has the information about the maximal blocks of $\mathbf{p}$. Applying a projectivity we can send three consecutive maximal blocks to $1, \infty$, and 0 . To define [p] completely, we have to choose $|\alpha|-3=: n$ real numbers such that $0<x_{1}<\cdots<x_{n}<1$ and this is an open simplex in $\mathbb{R}^{n}$.

The cells $\sigma_{\alpha} \subset \mathbb{P}_{k}^{1}$ behave as in a ball complex whose partial order is $\mathcal{D}_{k}$, because given $\alpha, \beta \in \mathcal{D}_{k}$ we have

$$
\alpha \preceq \beta \quad \Leftrightarrow \quad \sigma_{\alpha} \subseteq \overline{\sigma_{\beta}},
$$

where $\overline{\sigma_{\beta}}$ is the closure of $\sigma_{\beta}$. This follows because $\alpha \preceq \beta$ if and only if the blocks of $\alpha$ are obtained by joining consecutive segments of blocks of $\beta$ so that configurations in $\sigma_{\alpha}$ are limits of configurations in $\sigma_{\beta}$. The limits of configurations in $\sigma_{\beta}$ always have a coarser type. However, it is not a ball complex in the classic sense because of the non-Hausdorff phenomena-the closures of the cells are not closed balls.

If $\mathcal{R}$ is a good rule (satisfying R1-R3) we obtain a partial order $\mathcal{D}_{k}(\mathcal{R}) \subset \mathcal{D}_{k}$ of the dihedral partitions, all of whose blocks are permitted (in $\mathcal{R}$ ). We always adjoin to $\mathcal{D}_{k}(\mathcal{R})$ a new element 0 which is smaller than all others and a new element 1 which is bigger that all others. This new poset will be denoted the same way.

Lemma 3.2. If $\mathcal{R}$ is a good rule, then $\mathcal{D}_{k}(\mathcal{R})$ is a lattice.

Proof. Let us show that any two $\alpha, \beta \in \mathcal{D}_{k}(\mathcal{R})$ have a join $\alpha \vee \beta$. Suppose that there is a dihedral partition $\gamma$ such that $\gamma \succeq \alpha, \gamma \succeq \beta$. We can think of the blocks of $\alpha$ and $\beta$ as arcs in $\gamma$. If a block $A$ of $\alpha$ intersects a block $B$ of $\beta$, then $A \cap B$ is also an arc in 
$\gamma$ since otherwise $B \subset \bar{A}$, which is prohibited. Therefore we can construct $\gamma^{\prime} \preceq \gamma$ by joining in a block each arc in $\gamma$ which is an intersection of a block in $\alpha$ with a block in $\beta$. It is easy to check that $\gamma^{\prime}=\alpha \vee \beta$.

The proof concludes with the fact that any finite join semilattice with 0 is a lattice (see [2]).

Let $\theta$ be a dihedral order (a coatom) and let $\bar{\theta}=[0, \theta] \subset \mathcal{D}_{k}(\mathcal{R})$ be its closure. Denote by $T_{\theta}$ the poset obtained from $\bar{\theta} \backslash 0$ reversing the order relation.

Lemma 3.3. $\quad T_{\theta}$ is a pure simplicial complex of dimension $k-4$.

Proof. Denote by $E$ the set of pairs $\{i, j\}$ such that $i, j$ are consecutive in the dihedral order $\theta$. For $\alpha \in T_{\theta}$ we denote by $t_{\alpha}$ the set of all pairs $\{i, j\} \in E$ such that $i, j$ are in a same block of $\alpha$. Of course, $t_{\theta}=\emptyset$ and observe that $\alpha \preceq \beta$ iff $t_{\alpha} \subseteq t_{\beta}$. Moreover, if $A \subseteq t_{\beta}$, then there is $\alpha \in T_{\theta}$ such that $t_{\alpha}=A$ because we can always split the blocks of $\beta$ in the places defined by the elements of $E$. This means that $T_{\theta}$ is a simplicial complex. Finally, any maximal element $\alpha$ in $T_{\theta}$ has exactly three blocks and this implies that $\# t_{\alpha}=k-3$.

Now, recall (see Section 4.7 in [3]) that a shelling of a pure simplicial complex of dimension $d$ is a linear order $s_{1}, \ldots, s_{n}$ of its maximal simplices such that for all $1 \leq i<j \leq n$ there exist $1 \leq i^{\prime}<j$ such that $\operatorname{dim}\left(s_{i^{\prime}} \cap s_{j}\right)=d-1$ and $s_{i} \cap s_{j} \subseteq s_{i^{\prime}} \cap s_{j}$. A complex is said to be shellable if it has a shelling.

Lemma 3.4. If $\mathcal{R}$ is a good rule, then $T_{\theta}$ is shellable.

Proof. The notations in the proof of the preceding lemma are used. We choose one of the two orientations of the dihedral order $\theta$. We label an arbitrary element of $E$ by 1. The others elements of $E$ are labeled $2, \ldots, k$ by taking consecutive (in the chosen orientation) elements of $E$. With this labeling we have $E=\{1, \ldots, k\}$.

For any maximal simplex $\alpha \in T_{\theta}$ the set $E \backslash t_{\alpha}$ has exactly three elements of $E$ which we denote $\alpha_{1}<\alpha_{2}<\alpha_{3}$. Now, we can identify $\alpha$ with the vector $\left(\alpha_{1}, \alpha_{2}, \alpha_{3}\right)$. The set of all vectors $(a, b, c) \in E^{3}$ are linearly ordered by the lexicographic order and therefore the maximal simplices in $T_{\theta}$ are linearly ordered. We need to prove that it is a shelling.

Let $\alpha=\left(\alpha_{1}, \alpha_{2}, \alpha_{3}\right)<\left(\beta_{1}, \beta_{2}, \beta_{3}\right)=\beta$ be two maximal simplices in $T_{\theta}$. Define $\gamma=\left(\gamma_{1}, \gamma_{2}, \gamma_{3}\right)$ as:

- If $\alpha_{1}<\beta_{1}$, then (since the complement of a prohibited block is permitted) one of the two vectors $\left(\alpha_{1}, \beta_{2}, \beta_{3}\right),\left(\alpha_{1}, \beta_{1}, \beta_{2}\right)$ is a maximal simplex in $T_{\theta}$. In this case we put $\gamma$ equal to this simplex.

- If $\alpha_{1}=\beta_{1}$ and $\alpha_{2}<\beta_{2}$, then one of the two vectors $\left(\beta_{1}, \alpha_{2}, \beta_{3}\right),\left(\alpha_{2}, \beta_{2}, \beta_{3}\right)$ is a maximal simplex in $T_{\theta}$. In this case we put $\gamma$ equal to this simplex.

- If $\alpha_{1}=\beta_{1}$ and $\alpha_{2}=\beta_{2}$ then $\gamma=\alpha$. 
In all cases we have:

- $\gamma$ is a maximal simplex in $T_{\theta}$ and $\gamma<\beta$,

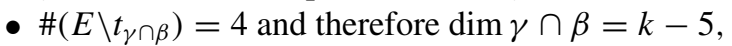

- $E \backslash t_{\gamma \cap \beta} \subset E \backslash t_{\alpha \cap \beta}=\left\{\alpha_{1}, \alpha_{2}, \alpha_{3}, \beta_{1}, \beta_{2}, \beta_{3}\right\}$ and therefore $\alpha \cap \beta \subseteq \gamma \cap \beta$,

and this is all we had to prove.

Lemma 3.5. If $\mathcal{R}$ is a good rule, then $T_{\theta}$ is the boundary complex of a convex polytope in $\mathbb{R}^{k-3}$.

Proof. Every simplex $\alpha \in T_{\theta}$ of dimension $k-5$ is contained in exactly two maximal simplices. Since $T_{\theta}$ is shellable, then by [5] it is $S^{k-4}$ (triangulated with at most $k$ vertices). By [9] all such triangulations are realizable as a convex polytope in $\mathbb{R}^{k-3}$.

Since $\mathcal{D}_{k}(\mathcal{R})$ is a lattice, then two maximal cells in $\mathcal{D}_{k}(\mathcal{R})$ intersect in a cell. Since the polars of maximal cells are convex polytopes and the polar of a convex polytope is a convex polytope itself, then we obtain for any good rule $\mathcal{R}$ the following:

Theorem 3.6. The lattice $\mathcal{D}_{k}(\mathcal{R})$ gives to $\mathbb{P}_{k}^{1}(\mathcal{R})$ the standard structure of a polyhedral complex.

Observe that the symmetric group $\mathfrak{S}_{k}$ acts naturally on $\mathcal{D}_{k}$, and accordingly on $\mathbb{P}_{k}^{1}$ preserving its cell structure. However, it usually sends a rule into another one, with one exception that should be noted. If $k$ is odd, then there is a special rule invariant under the action of $\mathfrak{S}_{k}$, the "choose the small" rule, $\mathcal{S}$, where a set $A$ is permitted iff \#A<k/2. Then $\mathbb{P}_{k}^{1}(\mathcal{S})$ with its polyhedral structure has symmetries $\mathfrak{S}_{k} ;$ Fig. 2 is the case $k=5$ with its cellular structure drawn.

\subsection{Attached Projective Cores}

Given an index set $A$, with $2 \leq \sharp A \leq k-2$, let $\mathbb{P}_{A}$ be the subspace of $\mathbb{P}_{k}^{1}$ consisting of all configurations that have maximal block $A$. The notation $\mathbb{P}_{A}$ comes about because $\mathbb{P}_{A}$ is homeomorphic to a projective space of dimension $k-a-2$, where $a=\sharp A$. To see this, observe that a configuration [p] with maximal block $A$ can be assumed to have its block $A$ at infinity, corresponding then to an affine configuration of the remaining indices $\bar{A}$ in the affine line because $\operatorname{Aff}(\mathbb{R})$ is the subgroup of PGL $\left(\mathbb{R}^{2}\right)$ that fixes infinity. That is, $\mathbb{P}_{A} \cong \mathbb{A}_{(k-a)}^{1}$, and this is homeomorphic to $\mathbb{P}^{k-a-2}$. Indeed, we may assume that the first point is zero, then equivalence is given by nonzero multiplication and the fixing hypothesis is that not all the points are zero.

Moreover, $\mathbb{P}_{A}$ is a subcomplex of $\mathbb{P}_{k}^{1}$ : it is the union of all the cells of dihedral partitions with block $A$. The poset of dihedral partitions with block $A$ is isomorphic to the poset of linear partitions of $\bar{A}$ which is a simplicial complex. Geometrically, it is the triangulation of $\mathbb{P}^{n}$, where $n=k-a-2$, obtained by the "chopping" of $\mathbb{P}^{n}$ by the hyperplanes spanned 
by $n+2$ points in general position (corresponding to the linear partitions $\{\{i\}, \bar{A} \backslash\{i\}\}$ ). For $n=2$, collapse the four inside circles of Fig. 2 to points.

By Proposition 2.3 we know that every point in $\mathbb{P}_{A}$ is attached to every point in $\mathbb{P}_{\bar{A}} \cong \mathbb{P}^{a-2}$. We will prove that, moreover, they have a common punctured regular neighborhood $V_{A}\left(=V_{\bar{A}}\right)$ homeomorphic to the standard punctured regular neighborhood of a projective flat in projective space.

To see this, we consider oriented configurations $\overrightarrow{\mathbb{P}}_{k}^{1}$, where we only divide by the orientation-preserving projectivities. We can give an explicit description of $S_{A}$ (the oriented double cover of $\mathbb{P}_{A}$, whose simplicial complex is the ordered partitions of $\bar{A}$ ) as

$$
S_{A}=\left\{[\mathbf{p}] \in \overrightarrow{\mathbb{P}}_{k}^{1}: \mathbf{p}_{A}=\{\infty\},\{0,1\} \subset \mathbf{p}_{\bar{A}} \subset[0,1]\right\},
$$

because for oriented affine configurations we have a well-defined minimum and maximum which can be assumed (via a unique orientation-preserving affinity) to be 0 and 1 , respectively. Now, a punctured regular neighborhood of $S_{A}$ can be given by the configurations [p] (and parametrized by the $k$-tuples $\mathbf{p}$ ) that satisfy the conditions

$$
\widetilde{V}_{A}:\left\{\begin{array}{l}
\{0,1\} \subset \mathbf{p}_{\bar{A}} \subset[0,1], \\
\{\infty\} \subset \mathbf{p}_{A} \subset[\infty,-1], \\
\sharp \mathbf{p}_{A} \geq 2
\end{array}\right.
$$

(where $[\infty,-1]$ is the interval from -1 to $\infty$ not containing 0 ), because a configuration $\mathbf{p}$ close to $S_{A}$ but not in $S_{A}$ has at least two different points in $A$ and the point (in $A$ ) closest to 1 may be projectively moved to $\infty$. Observe that if the third condition is deleted we obtain a (nonpunctured) regular neighborhood, with a natural (trivial) disk bundle structure (because the $A$-tuples $\{\infty\} \subset \mathbf{p}_{A} \subset[\infty,-1]$ are clearly homeomorphic to a disk $D^{a-1}$ ).

Analogously, changing the role of $A$ for $\bar{A}$, but not of the special points to make things explicit, we can describe $S_{\bar{A}}$ and $\widetilde{V}_{\bar{A}}$ as the configurations [p] that satisfy

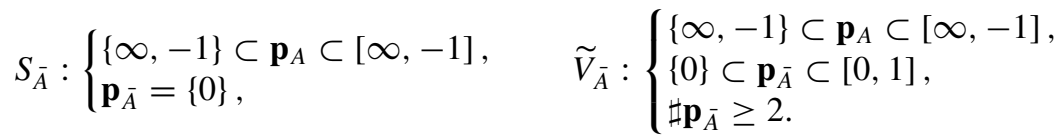

It is easy to see that $\widetilde{V}_{A} \equiv \widetilde{V}_{\bar{A}}$ because if $\mathbf{p}$ satisfies the conditions of $\tilde{V}_{\bar{A}}$ then $(1 / \max$ $\left.\left(\mathbf{p}_{\bar{A}}\right)\right)$ p satisfies those of $\widetilde{V}_{A}$. Then $\widetilde{V}_{A} \cup S_{A} \cup S_{\bar{A}}$ is homeomorphic to the quirurgical torus $H^{(k-a-2),(a-2)}$, with its boundary naturally homeomorphic to $S_{A} \times S_{\bar{A}}$ parametrized by the $k$-tuples $\mathbf{p}$ that satisfy $\{0,1\} \subset \mathbf{p}_{\bar{A}} \subset[0,1]$ and $\{\infty,-1\} \subset \mathbf{p}_{A} \subset[\infty,-1]$. Finally, observe that the orientation involution, given by the projectivity $x \mapsto(1-x) /(x+1)$, acts as the product of the antipodal maps so that when passing to the quotient, the nearby cores $\mathbb{P}_{A}$ and $\mathbb{P}_{\bar{A}}$ have the standard regular neighborhoods of complementary projective flats in $\mathbb{P}^{k-3}$, which coincide when punctured.

\subsection{Surgery}

Projective surgery is what we call the replacement in a manifold $M^{m}$ of a submanifold homeomorphic to $\mathbb{P}^{n}$ with normal bundle equivalent to the standard one (that of $\mathbb{P}^{n} \hookrightarrow$ $\left.\mathbb{P}^{m}\right)$ by its complementary projective space $\mathbb{P}^{m-n-1}$. We will prove the following. 
Theorem 3.7. The full-dimensional submanifolds of $\mathbb{P}_{k}^{1}$ can be obtained from each other by projective surgery staying within $\mathbb{P}_{k}^{1}$.

Proof. By Theorem 2.1 we might as well analyze the good rules $\mathcal{R}$ (satisfying R1$\mathrm{R} 3$ ). Suppose that $\mathcal{R}$ is a good rule and that $A$ is a maximal permitted block in $\mathcal{R}$ with $2 \leq \sharp A \leq k-2$. We claim that $\mathbb{P}_{A} \subset \mathbb{P}_{k}^{1}(\mathcal{R})$, this is, that all the cells whose dihedral order have $A$ as a block are in $\mathbb{P}_{k}^{1}(\mathcal{R})$. To see this, suppose $\alpha \in \mathcal{D}_{k}$ has a block $A$. Suppose $B$ is another block of $\alpha$, since $A \varsubsetneqq \bar{B}$ and $A$ is maximal in $\mathcal{R}$, then $\bar{B}$ is prohibited and hence $B$ is permitted. Therefore, $\sigma_{\alpha} \subset \mathbb{P}_{k}^{1}(\mathcal{R})$, which proves that $\mathbb{P}_{A} \subset \mathbb{P}_{k}^{1}(\mathcal{R})$. Performing projective surgery along $\mathbb{P}_{\bar{A}}$ is replacing it by $\mathbb{P}_{\bar{A}}$ and the manifold we obtain is $\mathbb{P}_{k}^{1}\left(\mathcal{R}^{\prime}\right)$, where $\mathcal{R}^{\prime}=(\mathcal{R} \backslash\{A\}) \cup\{\bar{A}\}$, that is, $A$ becomes prohibited and $\bar{A}$ permitted.

We are left to see that we can go from any good rule to another by exchanges of this type. For this, we consider the affine rule $\mathcal{A}$, with permitted blocks (in $\mathcal{A}$ ) all singletons and subsets without $k$, except $\frac{j}{\{k\}}$. So, taking $p_{k}=\infty$, we have $\mathbb{P}_{k}^{1}(\mathcal{A})=\mathbb{A}_{k-1}^{1} \cong \mathbb{P}^{k-3}$ with the triangulation of linear partitions of $\overline{\{k\}}$. Given any good rule $\mathcal{R}$, denote by $\mathcal{R}^{i}$ the set of permitted blocks in $\mathcal{R}$ with $i$ elements. For each $A \in \mathcal{R}^{2} \backslash \mathcal{A}$ (permitted 2-blocks of $\mathcal{R}$ containing $k$ ) we have that $\bar{A} \in \mathcal{A}$ and can exchange it as above (in this case it corresponds to the blow-up at a distinguished vertex of $\mathbb{P}_{k}^{1}(\mathcal{A})$ ); to get a new good rule $\mathcal{R}_{2}$ coinciding with $\mathcal{R}$ in blocks of order 2 , so that $\mathbb{P}_{k}^{1}\left(\mathcal{R}_{2}\right)$ is obtained from $\mathbb{P}_{k}^{1}(\mathcal{A})$ by projective surgery on points. Then, one can exchange all the blocks $A \in \mathcal{R}^{3} \backslash \mathcal{A}$ (surgery on projective lines), and so on through $\mathcal{R}^{4}, \ldots$ to get finally to $\mathcal{R}$. This clearly completes the proof of the theorem.

\section{Duality and Polarity}

In this section we return to the general case when $\mathbb{K}$ is any field.

\subsection{Polarity}

We first describe the polarity between projective configurations of flats (we reserve the term "duality" for another map). There is a natural homeomorphism

$$
\mathbb{K P}_{\mathbf{d}}^{n} \longleftrightarrow \mathbb{K P}_{n-\mathbf{d}-1}^{n}
$$

that arises because each flat $F$ in $\mathbb{K} \mathbb{P}^{n}$ has a polar $F^{\perp}$. More precisely, $F$ corresponds to a linear subspace $\xi$ in $\mathbb{K}^{n+1}$, consider its orthogonal complement $\xi^{\perp}$, and then its corresponding flat $F^{\perp}$ in $\mathbb{K P}^{n}$ is the polar of $F$. So, to a $k$-tuple of flats $F_{1}, \ldots, F_{k}$ $\left(\operatorname{dim} F_{i}=d_{i}\right)$ there corresponds a polar $k$-tuple of flats $F_{1}^{\perp}, \ldots, F_{k}^{\perp}\left(\operatorname{dim} F_{i}=n-d_{i}-\right.$ $1)$. We still have to consider the action of PGL $\left(\mathbb{K}^{n+1}\right)$ on these $k$-tuples. It happens that the two actions correspond to each other, so that polarity goes down to the quotients (1). Consider $A \in \mathrm{GL}\left(\mathbb{K}^{n+1}\right)$ and $\xi$ a linear subspace of $\mathbb{K}^{n+1}$, then a standard linear algebra calculation yields

$$
(A \xi)^{\perp}=\left(A^{\top}\right)^{-1} \xi^{\perp}
$$


So that if two $k$-tuples of flats are projectively equivalent, by means of a matrix $A$ say, then their polar $k$-tuples are also projectively equivalent, but by means of the inverse of the transpose of $A$.

\subsection{Duality}

What we call "duality" follows the general idea first exposed by Whitney [10] for the duality of matroids (see also [6]). It goes, briefly, as follows. Consider a $k$-tuple of points $\mathbf{x}=\left(x_{1}, \ldots, x_{k}\right) \in\left(\mathbb{K}^{n}\right)^{k}$ that linearly span $\mathbb{K}^{n}$. The classes of these $k$-tuples modulo the diagonal action of GL( $\left.\mathbb{K}^{n}\right)$ are called vector configurations. The space of all vector configurations will be denoted by $\mathbb{K}_{k}^{n}$. Observe that we can replace $\mathbb{K}^{n}$ by any other vector space of dimension $n$ over $\mathbb{K}$, since we are dividing by the general linear group and, therefore, there is a canonical isomorphism. Let $e_{1}, \ldots, e_{k}$ be the canonical base of $\mathbb{K}^{k}$. The map

$$
G_{k-n}\left(\mathbb{K}^{k}\right) \ni \theta \mapsto\left(e_{1}+\theta, \ldots, e_{k}+\theta\right) \in\left(\mathbb{K}^{k} / \theta\right)^{k}
$$

induces a homeomorphism $G_{k-n}\left(\mathbb{K}^{k}\right) \leftrightarrow \mathbb{K}_{k}^{n}$. Composing it with the "orthogonal complement" $G_{k-n}\left(\mathbb{K}^{k}\right) \leftrightarrow G_{n}\left(\mathbb{K}^{k}\right)$ homeomorphism we obtain the vector configuration duality homeomorphism $\mathbb{K}_{k}^{n} \leftrightarrow \mathbb{K}_{k}^{k-n}$.

Now, for configurations of flats, recall that $\mathbb{K P}_{\mathbf{d}-1}^{n-1}$ is the space of all configurations of projective flats $\left[F_{1}, \ldots, F_{k}\right]$ in $\mathbb{K} \mathbb{P}^{n-1}$ such that $\operatorname{dim} F_{i}=d_{i}-1$ where $\mathbf{d}=\left(d_{1}, \ldots, d_{k}\right)$. We identify $F_{i}$ with its corresponding $d_{i}$-dimensional linear subspace in $\mathbb{K}^{n}$. Denote $|\mathbf{d}|=\sum d_{i}$. Let $E_{1}$ be the subspace of $\mathbb{K}^{|\mathbf{d}|}$ spanned by the first $d_{1}$ vectors of the canonical basis $e_{1}, \ldots, e_{|\mathbf{d}|} ; E_{2}$ the subspace of $\mathbb{K}^{|\mathbf{d}|}$ spanned by the following $d_{2}$ vectors of the canonical basis and so on. Finally, denote by $\Gamma_{\mathbf{d}}$ the subgroup of PGL( $\left(\mathbb{K}^{|\mathbf{d}|}\right)$ which leaves each of the subspaces $E_{1}, \ldots, E_{k}$ invariant, i.e., $\Gamma_{\mathbf{d}}=\prod_{i} \operatorname{PGL}\left(E_{i}\right)$.

Lemma 4.1. There is an embedding $\mathbb{K P}_{\mathbf{d}-1}^{n-1} \hookrightarrow G_{|\mathbf{d}|-n}\left(\mathbb{K}^{|\mathbf{d}|}\right) / \Gamma_{\mathbf{d}}$ whose image is (modulo $\left.\Gamma_{\mathbf{d}}\right)$ the open subset of $G_{|\mathbf{d}|-n}\left(\mathbb{K}^{|\mathbf{d}|}\right)$ where the action of $\Gamma_{\mathbf{d}}$ is free.

Proof. For any $\theta \in G_{|\mathbf{d}|-n}\left(\mathbb{K}^{|\mathbf{d}|}\right)$ such that $\theta \cap E_{i}=0$ the subspace $E_{i}+\theta$ of $\mathbb{K}^{|\mathbf{d}|} / \theta$ has dimension $d_{i}$. Observe that if these subspaces fix, then any isomorphism $\mathbb{K}^{k} / \theta \leftrightarrow \mathbb{K}^{n}$ gives the same configuration in $\mathbb{K} \mathbb{P}_{\mathbf{d}-\mathbf{1}}^{n-1}$. So, we have a partial map

$$
G_{|\mathbf{d}|-n}\left(\mathbb{K}^{|\mathbf{d}|}\right) \ni \theta \mapsto\left[E_{1}+\theta, \ldots, E_{k}+\theta\right] \in \mathbb{K}_{\mathbf{d}-\mathbf{1}}^{n-1}
$$

and two spaces $\theta, \theta^{\prime}$ have the same image iff $\theta=\theta^{\prime} \bmod \Gamma_{\mathbf{d}}$.

Now, for $f \in \Gamma_{\mathbf{d}}$ we have $f(\theta)=\theta$ iff $f\left(E_{i}+\theta\right)=E_{i}+\theta$. If

$$
\left[E_{1}+\theta, \ldots, E_{k}+\theta\right] \in \mathbb{K P}_{\mathbf{d}-1}^{n-1}
$$

then $f$ must be the identity (since $E_{1}+\theta, \ldots, E_{k}+\theta$ fix $\mathbb{K}^{|\mathbf{d}|} / \theta$ ). Finally, if $\theta \cap E_{i} \neq 0$ for some $i$, then there are nonidentity maps in $\operatorname{PGL}\left(E_{i}\right)$ which leave invariant $\theta$.

Remark 4.1. The above lemma shows that, in fact, we are studying quotients of open subsets of Grassmannians by groups of block diagonal matrices. In the case that all 
blocks are of dimension 1, the group $\Gamma_{\mathbf{d}}$ is a maximal torus in $\mathrm{GL}\left(\mathbb{C}^{|\mathbf{d}|}\right)$, the lemma is a result in [6] and the Chow quotients of $G_{n}\left(\mathbb{C}^{|\mathbf{d}|}\right)$ by $\Gamma_{\mathbf{d}}$ are studied in [8].

To obtain the duality homeomorphism it only remains to observe that the group $\Gamma_{\mathbf{d}}$ is closed by taking transposes and (2) shows that the stabilizer of $\theta$ by the action of $\Gamma_{\mathbf{d}}$ in $G_{|\mathbf{d}|-n}\left(\mathbb{K}^{|\mathbf{d}|}\right)$ is isomorphic (taking the inverse of the transpose) to the stabilizer of $\theta^{\perp}$ by the action of $\Gamma_{\mathbf{d}}$ in $G_{n}\left(\mathbb{K}^{|\mathbf{d}|}\right)$.

We summarize our results changing the indices to the standard ones.

Theorem 4.2. There is a natural duality $\mathbb{K}_{\mathbf{d}}^{n} \longleftrightarrow \mathbb{K P}_{\mathbf{d}}^{m}$ where

$$
m=\sum\left(d_{i}+1\right)-n-2 .
$$

\subsection{An Application}

If we iterate duality and polarity we get infinite families of equivalences. For example, the first three members of the sequence

$$
\mathbb{P}_{5,0}^{1}=\mathbb{P}_{5,0}^{2}=\mathbb{P}_{5,1}^{2}=\mathbb{P}_{5,1}^{6}=\mathbb{P}_{5,4}^{6}=\mathbb{P}_{5,4}^{17}=\mathbb{P}_{5,12}^{17}=\mathbb{P}_{5,12}^{46}=\cdots
$$

appeared in the Introduction.

In the paper we fully described $\mathbb{P}_{k, 0}^{1}$ and therefore we also fully described all the spaces obtained by alternating polarity and duality. For the affine case the arguments in the Introduction can be generalized to describe

$$
\mathbb{A}_{n+2, n-1}^{n} \subset \mathbb{P}_{n+3, n-1}^{n}=\mathbb{P}_{n+3,0}^{n}=\mathbb{P}_{n+3,0}^{1}
$$

and to prove that $\mathbb{A}_{n+2, n-1}^{n}(\neq)$ is $\mathbb{P}^{n}$ with $n+2$ blowups.

\section{References}

1. J. L. Arocha, J. Bracho, L. Montejano, D. Oliveros, and R. Strausz. Separoids, their category and a Hadwiger type theorem for transversals. Discrete Comput. Geom. 23:377-385, 2002.

2. G. Birkhoff. Lattice Theory, 3rd edn. Volume 25 of American Mathematical Society Colloquium Publications. American Mathematical Society, Providence, R.I., 1979.

3. A. Björner, M. Las Vergnas, B. Sturmfels, N. White, and G. Ziegler. Oriented Matroids, 2nd edn. Volume 46 of Encyclopedia of Mathematics and its Applications. Cambridge University Press, Cambridge, 1999.

4. J. Bracho, L. Montejano, and D. Oliveros. The topology of the space of transversals through the space of configurations. Topology Appl., 120(1-2):93-103, 2002. In memory of T. Benny Rushing.

5. G. Danaraj and V. Klee. Shellings of spheres and polytopes. Duke Math. J., 41:443-451, 1974.

6. I. M. Gel'fand, R. M. Goresky, R. D. MacPherson, and V. V. Serganova. Combinatorial geometries, convex polyhedra, and Schubert cells. Adv. Math., 63(3):301-316, 1987.

7. I. Hubard. Poliedros coloreados con órdenes cíclicos. B.Sc. Thesis, Fac. Ciencias, UNAM, 2001.

8. M. M. Kapranov. Chow quotients of Grassmannians, I. In I. M. Gel'fand Seminar, pages 29-110. Volume 16 of Advances in Soviet Mathematics. American Mathematics Society, Providence, RI, 1993.

9. P. Mani. Spheres with few vertices. J. Combin. Theory Ser. A, 13:346-352, 1972.

10. H. Whitney. On the abstract properties of linear dependence. Amer. J. Math., 57:509-533, 1935.

Received March 13, 2003, and in revised form August 16, 2004. Online publication April 15, 2005. 